\title{
Dissociation between adipose tissue signals, behavior and the food-entrained oscillator
}

\author{
M T Martínez-Merlos, M Ángeles-Castellanos, M Díaz-Muñoz ${ }^{1}$, \\ R Aguilar-Roblero², J Mendoza and C Escobar \\ Departamento de Anatomía, Facultad de Medicina, Universidad Nacional Autónoma de México, Mexico \\ ${ }^{1}$ Departmento de Neurobiología Celular y Molecular, Instituto de Neurobiología, Juriquilla, Universidad Nacional Autónoma de México, Mexico \\ ${ }^{2}$ Departamento de Neurociencias, Instituto de Fisiología Celular, Universidad Nacional Autónoma de México, Mexico \\ (Requests for offprints should be addressed to C Escobar; Email: cescobar@ifc.unam.mx)
}

\begin{abstract}
Digestive and metabolic processes are entrained by restricted feeding (RFS) schedules and are thought to be potential elements of a food-entrained oscillator (FEO). Due to the close relationship of leptin with metabolic regulation and because leptin is a relevant communication signal of the individual's peripheral metabolic condition with the central nervous system, we explored whether leptin is an endogenous entraining signal from the periphery to a central element of an FEO. First we characterized in the rat the diurnal rhythm of serum leptin (in rats fed ad libitum (AL)), its adjustment to an RFS and the influence of fasting after RFS, or RFS followed by AL feeding and then total food deprivation (RF-AF) in the persistence of this fluctuating pattern. We also explored the response of free fatty acids and stomach weight under the same entraining conditions. We compared the metabolic response with the behavioral expression of drinking anticipatory activity (AA) under the same conditions. Finally, we tested the effect of daily i.c.v administration of leptin as a putative entraining signal for the generation of AA.
\end{abstract}

Metabolic parameters responded to food entrainment by adjusting their phase to mealtime. However, leptin and free fatty acid rhythms persisted only for a few cycles in fasting conditions and readjusted to the light-darkness cycle after an RF-AF protocol. In contrast, behavioral food-entrained rhythms persisted after both fasting manipulations. Daily leptin i.c.v. administration did not produce AA, nor produce changes in the behavioral free-running rhythm. Stomach weight indicated an adaptive process allowing an extreme stomach distension followed by a slow emptying process, which suggests that the stomach may be playing a relevant role as a storage organ.

In conclusion, metabolic signals here studied respond to feeding schedules by adjusting their phase to mealtime, but do only persist for a few cycles in fasting. Leptin does not produce AA and thus is not an entraining signal for FEO. The response of metabolic signals to feeding schedules depends on different mechanisms than the expression of AA.

Journal of Endocrinology (2004) 181, 53-63

\section{Introduction}

Rats maintained under restricted feeding schedules (RFS) show a phase shift towards mealtime in the peak of plasma corticosterone, body temperature levels (Krieger 1974, Honma et al. 1984) and in serum energy metabolites, as well as in glucagon and insulin concentration (Escobar et al. 1998, Díaz-Muñoz et al. 2000). Metabolic food entrainment is associated with the expression of behavioral anticipatory activity (AA), characterized by increased locomotor activity starting $2-3 \mathrm{~h}$ previous to the mealtime. Also RFS entrain the expression of clock genes in peripheral tissues while leaving unaffected the phase of cyclic gene expression in the suprachiasmatic nuclei (SCN) of the hypothalamus (Damiola et al. 2000).
Despite physiological and behavioral evidence of the expression of a food-entrained oscillator (FEO), its anatomical location as well as its mechanisms are not well understood (Stephan 2002). Because lesions of several nervous structures do not interfere with AA (Mistlberger 1994, Davidson \& Stephan 1998), nor with food-entrained rhythmicity in peripheral tissues (Krieger et al. 1977, Honma et al. 1987, Davidson \& Stephan 1999) we propose that the FEO might consist of a distributed system involving central nervous structures and peripheral organs forming feedback loops. In this model, communication between central and peripheral elements could be accomplished through humoral signals or synaptic connections. Because many digestive processes are entrained to mealtime, it is possible that physiological or metabolic signals 
elicited by digestive organs are elements of the internal entraining pathway to produce a meal-induced signal toward the central elements of the FEO.

Recent studies explored peripheral catecholamines as a possible internal entraining signal for peripheral clocks and reported that arrhythmic SCN-lesioned rats exposed to daily injections of adrenaline for several days recovered oscillations of clock genes in the liver (Terazono et al. 2003). Also, we reported that daily injections of adrenaline in intact rats produced behavioral activation similar to food AA (Mendoza et al. 2003). Because the intensity of this behavioral activation was significantly lower than that observed during food anticipation, we suggest that catecholamines may not be the only internal entraining signal elicited by food to the FEO.

Another possible peripheral element in this distributed model of an FEO may be the white adipose tissue. In a previous communication we reported that serum free fatty acids (FFA), one of its products, are entrained by RFS (Escobar et al. 1998). In addition, its hormone leptin has shown to be a relevant communication signal of the individual's peripheral metabolic condition to the central nervous system (Campfield et al. 1995, Schwartz et al. 2000) and thus may be part of the endogenous entraining signal from the periphery to a central element of the FEO. Leptin is released from the white adipose tissue in proportion to adiposity (Zhang et al. 1994, Ahima \& Flier 2000), decreases under fasting conditions (Dallongeville et al. 1998) and is secreted at a higher rate following food ingestion. Low doses of leptin injected into the brain produce a satiety response (Campfield et al. 1995, Halaas et al. 1997), which includes a decrease in exploratory behavior (Buyse et al. 2001) and reduction of meal size in rats (Kahler et al. 1998, Ruffin \& Nicolaidis 2000). The main effects of leptin are mediated through its specific receptors that are highly expressed in hypothalamic nuclei (Ahima 2000), particularly in the arcuate, dorsomedial, ventromedial and ventral premammillary nuclei (Friedman 2000, Elmquist 2001).

Leptin levels in serum show diurnal variations in humans (Sinha et al. 1996) and mice (Ahima et al. 1998) associated with the dark period and are entrained to mealtime (Elimam \& Marcus 2002). The relationship of leptin as a peripheral signal with the main circadian clock, the SCN, was recently explored in vitro. Leptin, applied in the perfusion medium of SCN slices at different times across the $24 \mathrm{~h}$ cycle, produced phase advances in the SCN electrical activity in a dose-dependent manner (Prosser \& Bergeron 2003). Because the observed phase shifts were of lower magnitude than those observed with the injection of other substances, it was suggested that another oscillator, for instance the FEO, could mediate the main effect.

Despite the close relationship of leptin with metabolic regulation, the relationship of leptin with the FEO's mechanisms has not been explored. In particular the role of leptin as an endogenous food-entraining signal from the periphery to the brain has not been established. Thus, the aim of the present study was to determine the relationship of products of white adipose tissue with the FEO. First we characterized in the rat the diurnal rhythm of serum leptin and its adjustment to feeding schedules. We aimed to explore the role of serum leptin as a putative entraining signal by determining its adjustment to an RFS, the influence of fasting on this fluctuating pattern and the effect of i.c.v. administration of leptin in the generation of AA.

\section{Materials and Methods}

\section{Animals and general housing conditions}

Adult male Wistar rats (250-320 g at the beginning of the experiment) were maintained in a $12 \mathrm{~h}$ light: $12 \mathrm{~h}$ darkness cycle $(\mathrm{ZT0}=$ lights on at $0700 \mathrm{~h})$, constant temperature $\left(22 \pm 1{ }^{\circ} \mathrm{C}\right)$ and free access to food (Rodent Laboratory Chow 5001, Agribrands Purina, Canada) and water, unless otherwise stated. Experimental procedures were approved and conducted according to the institutional guide for care and use of animal experimentation (Universidad Nacional Autónoma de México), which conforms with the guidelines of the UFAW Handbook on the Care and Management of Laboratory Animals (UK).

\section{Experiment I}

Experiment I was aimed at characterizing diurnal and food-entrained rhythms in serum leptin, FFA and stomach weight and the persistence of food-entrained rhythms in constant fasting conditions.

To determine daily and food-entrained rhythmicity, rats were randomly assigned for 21 days to one of two feeding conditions: controls fed ad libitum (AL), in which animals had free access to food and water during the $24 \mathrm{~h}$ cycle, and restricted feeding (RF), in which food access was restricted to $2 \mathrm{~h}$ daily from 1200 to $1400 \mathrm{~h}$ (ZT5-ZT7).

In order to determine persistence of entrained rhythms a second set of rats was randomly assigned to one of two fasting conditions: RF for 21 days, followed by total food deprivation for 2 days (RF-F), and RF for 21 days, followed by 1 week AL feeding and 2 days of total food deprivation (RF-AF). This procedure allowed us to observe persistence of food-entrained rhythms for 9-10 days.

At the end of each feeding protocol, animals were weighed and serum and tissue samples were obtained every $3 \mathrm{~h}(n=8)$ starting at $0900 \mathrm{~h}$ to complete a $24 \mathrm{~h}$ cycle (ZT2, ZT5, ZT8, ZT11, ZT14, ZT17, ZT20 and ZT23). The sampling of RF-F and RF-AF rats started after $43 \mathrm{~h}$ of fasting (geographical time $0900 \mathrm{~h}$ or ZT2) to complete a $24 \mathrm{~h}$ cycle, which represented $64 \mathrm{~h}$ of fasting (ZT23). 
A third set of rats was used to monitor behavior in $\mathrm{AL}$ and RF animals. Animals were also randomly assigned to an RF-F $(n=8)$ or an RF-AL $(n=8)$ protocol to determine persistence of $\mathrm{AA}$ in both fasting conditions.

Serum and tissue sampling For each temporal point and each group, seven or eight rats were killed by beheading and trunk blood (3-4 ml) was collected in $10 \mathrm{ml}$ test tubes containing a 'clot-forming gel' (Vacutainer; Becton Dickinson, NJ, USA). Tubes were centrifuged at $2500 \mathrm{~g}$ for $15 \mathrm{~min}$ to obtain blood serum. Aliquots of 250 and $700 \mu \mathrm{l}$ were coded and frozen at $-70{ }^{\circ} \mathrm{C}$ for subsequent determination of leptin and FFA concentrations.

After blood collection, the stomach was dissected at the level of the lower gastroesophageal sphincter and the pylorus and wet stomach weight was registered. The retroperitoneal and epididymal adipose tissue were bilaterally dissected and weighed according to Bartness (1987).

Determination of serum leptin and FFA Serum leptin was determined in duplicate with a standard ${ }^{125}$ I RIA kit (Linco Research, Inc., St Charles, MO, USA). Leptin assays were performed with a sensitivity of $0.5 \mathrm{ng} / \mathrm{ml}$ and intra- and interassay coefficients of variation of $4 \%$.

Serum FFA were processed by using a colorimetric method (Novák 1965), as described elsewhere (Escobar et al. 1998).

Behavioral monitoring Rats were placed in individual cages $(50 \times 30 \times 35 \mathrm{~cm})$ in a soundproof room with a regulated temperature of $22 \pm 1{ }^{\circ} \mathrm{C}$ and constant dim red light $(25 \mathrm{~lx})$. The individual drinking behavior was monitored continuously with an automated system. Number of touches to the water spout were stored in 15 min bins in a computer and saved data were visualized as double plot actograms using a digital analysis system applied to chronobiology (DiSPAC) automatic system for drinking behavior (Aguilar-Roblero et al. 1997). Animals were monitored for 15 days with free access to food and water; on day 16 food was removed and rats were maintained for 14-20 days under an RFS, with food access from 1200-1400 h local time. For the RF-F group this was followed by 5 days of fasting, while for the RF-AL this was followed by 10 days of free food access and 5 days of fasting (food was removed at $1400 \mathrm{~h}$ ), in order to determine persistence of AA. With the mean activity for each $15 \mathrm{~min}$ time point individual activity waveforms were obtained for the intervals of food-entrainment and for fasting. Graphic display of actograms and activity waveforms were obtained with the DiSPAC (Aguilar-Roblero et al. 1997).

Statistical analysis Data are reported as means \pm S.E.M. and are represented as temporal waveforms. Body weight before and after each feeding protocol was compared with a one-way ANOVA. Differences between AL and RF groups were tested with a two-way ANOVA for the main factors feeding schedule $\times$ time. Data for the fasted groups were analyzed with a one-way ANOVA to identify main effects due to the time factor. All ANOVAs were followed by a Tukey multiple comparison post hoc test with $\alpha$ set at $P<0 \cdot 05$. Correlation between leptin concentration and adipose tissue weight was obtained with a Pearson correlation coefficient. Statistical analysis was performed with the package Statistica version 4.3 (StatSoft, Inc. 1993).

\section{Experiment II}

The second experiment was aimed to examine the role of leptin as an entraining signal towards a central component of an FEO and therefore we explored the capability of daily i.c.v. leptin administration to produce behavioral activation similar to the AA.

Experimental design Free-running rhythms of drinking activity were monitored in rats bearing a fixed cannula above the third ventricle. After 15 days of free-running baseline the effect of daily leptin or vehicle injections on rhythmicity were assessed in a counterbalanced design. A first subgroup of rats received during 10 days an i.c.v. injection of leptin at local time $1400 \mathrm{~h}($ Leptin $\mathrm{ZT}=0)$ and a second subgroup of animals received at the same time an injection of vehicle. The treatment was followed by another 10 days in free-running conditions. Rats received then in a counterbalanced manner either leptin or vehicle for another 10 days followed by 10 days of free-running conditions.

i.c.v. cannulation Rats were anesthetized with an i.p. injection of chloral hydrate ( $40 \mathrm{mg} / 100 \mathrm{~g}$ body weight). Rats received an implanted 21-gauge guide cannula above the third ventricle according to stereotaxic coordinates: $\mathrm{AP}=-2 \cdot 3, \mathrm{~L}=0$, with respect to bregma (Paxinos \& Watson 1986), and $V=-7 \mathrm{~mm}$ from the skull surface. The cannula was fixed to the skull with four stainless steel screws and dental acrylic. An 18-gauge stylet was introduced into the guide cannula to maintain its permeability. Rats were allowed to recover from the surgery for 1 week before starting behavioral monitoring.

To have an indirect indicator of the cannula placement we tested whether i.c.v. leptin administration induced an inhibitory effect over the amount of food intake. We monitored food intake for a $24 \mathrm{~h}$ cycle, injected i.c.v. leptin $(2 \mu \mathrm{g} / 2 \mu \mathrm{l})$ and monitored food intake for another $24 \mathrm{~h}$ cycle. Food intake was reduced by $20 \%$ in leptininfused rats, which is in agreement with previous results (Flynn et al. 1998, Wang et al. 1999).

Animals were then placed in individual cages for automatic behavioral monitoring as previously described.

Leptin or vehicle injections Leptin (Linco) was dissolved in sterile saline solution $(0.9 \%)$ at a concentration of 
$1 \mu \mathrm{g} / \mu \mathrm{l}$. For daily leptin i.c.v. administration the injector cannula (27-gauge) was attached to a $5 \mu$ l Hamilton $26 \mathrm{~mm}$ syringe, with an $0 \cdot 01$ inch internal diameter silicon tubing (Tygon, IL, USA). The injector cannula was flushed with sterile saline followed by leptin or vehicle solution. Rats were gently handled; the stylet was removed from the guide cannula to insert the injector and $2 \mu \mathrm{g}$ leptin in $2 \mu \mathrm{l}$ vehicle were injected during an interval of 40-60 s, after which the injection cannula was left in place for an additional 40-60 s before replacing the stylet. The vehicle group received $2 \mu \mathrm{l}$ sterile saline solution as described for leptin.

Histology At the end of the experiment, rats were profoundly anesthetized with sodium pentobarbital and transcardially perfused with physiological saline solution $(\mathrm{NaCl}, 0.9 \%)$ followed by $10 \%$ formalin. Brains were removed and stored in 10\% formalin and cryoprotected with sucrose (10, 20 and 30\%). Frozen sections were cut in $40 \mu \mathrm{m}$ sections through the placement area of the cannula, mounted on slides and stained with cresyl violet (Nissl), dehydrated with alcohol, cleared with xylene and cover slipped. Brains were inspected with a Nikkon light microscope (E600, Técuica en laboratories, SA, Mexico) with $4 \times$ magnification to verify placement of the cannula. Data from rats in which the tip of the cannula was found close to but outside of the third ventricle (anterior hypothalamic area and dorsomedial nucleus) or rats that lost the stylets during the experiment were discarded from further statistical analysis. Finally behavioral data from eight rats were included for the analysis.

Analysis of behavioral data Data from drinking behavior are represented as actograms and average waveforms of mean spout licking/15 min bins for 10 days. Periods for the 10 day intervals during baseline, after leptin and vehicle injections were obtained with a $\chi^{2}$-periodogram using the program DiSPAC. Differences between groups were analyzed with a two-way ANOVA for the main factors feeding schedule $\times$ time (repeated measures), followed by a Tukey multiple-comparison post hoc test, with $\alpha$ set at $P<0 \cdot 05$.

\section{Results}

\section{Experiment I}

Rats fed AL exhibited an increase of $44 \%$ with respect to their initial body weight, while rats entrained to RFS gained $13 \%$ of body weight, RF-F showed no increase and RF-AF 14\%. The one-way ANOVA indicated a significant effect due to feeding condition $\left(\mathrm{F}_{(3,243)}=120 \cdot 4\right.$, $P<0 \cdot 001)$. The Tukey post hoc test confirmed that the AL group was significantly different from all groups $(P<0 \cdot 0001)$ and RF and RF-AF were different from the RF-F group $(P<0 \cdot 0001)$.

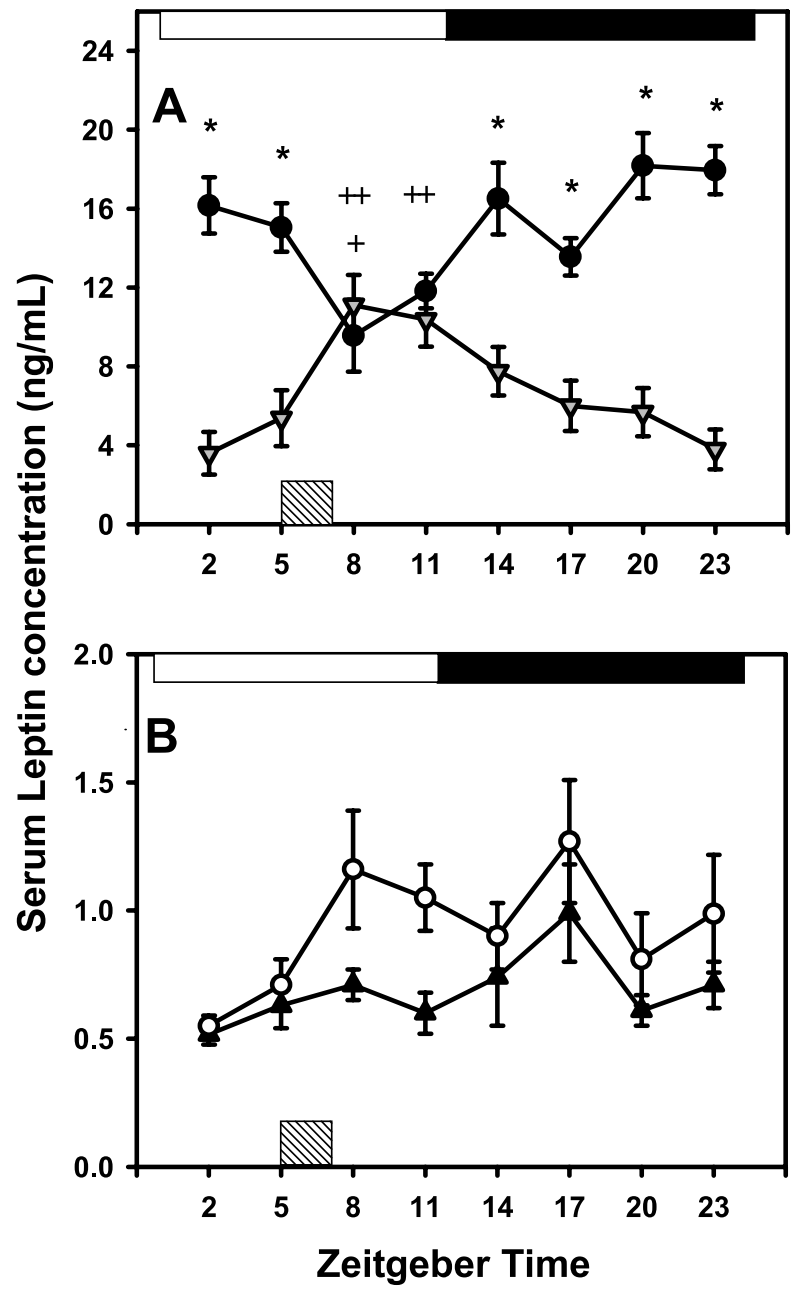

Figure 1 (A) Mean serum leptin diurnal rhythm (means \pm S.E.M.) in $\mathrm{AL}$ rats (black circles) and its entrainment in RF rats (gray triangles). (B) Serum leptin rhythms in fasting conditions after food entrainment (RF-F, white circles) and after a food entrainment-AF protocol (black triangles). For each group $n=8$ at each time point. The food-entrained pattern did not persist under fasting conditions, although a low-amplitude peak can still be observed in the RF-F group. White and black bars above indicate lighting conditions. * Difference between $\mathrm{AL}$ and $\mathrm{RF}, P<0 \cdot 01$; ${ }^{+}$significant difference in the AL group between ZT8 and other time points $P<0 \cdot 01) .{ }^{++}$Significant difference in RF ZT8 and ZT11 vs ZT5, ZT2 and ZT23, $P<0.01$ (Tukey post hoc test). The bar on the abscissa indicates mealtime or expected mealtime in fasting conditions.

Diurnal leptin rhythm A clear diurnal rhythm in serum leptin was observed in AL rats, with peak values at ZT20, the lowest values at ZT8 (Fig. 1A) and mean daily concentration of $15.5 \mathrm{ng} / \mathrm{ml}$. This diurnal rhythm was entrained to the RFS, characterized by a phase shift of rhythmicity towards mealtime. The RF group exhibited peak values after feeding at ZT8, the lowest values prior to food access (ZT23 and ZT2) with a mean daily leptin concentration of $6.5 \mathrm{ng} / \mathrm{ml}$. The two-way ANOVA 
revealed a significantly main effect between groups $\left(\mathrm{F}_{(1,112)}=165 \cdot 81, P<0 \cdot 0001\right)$, no significant difference due to time $\left(\mathrm{F}_{(7,112)}=1 \cdot 0, \quad \mathrm{NS}\right)$, but a significant group $\times$ time interaction $\left(\mathrm{F}_{(7,112)}=9 \cdot 6, P<0 \cdot 0001\right)$.

The average serum leptin concentration was importantly decreased in fasted animals: $0.93 \pm 0.07 \mathrm{ng} / \mathrm{ml}$ for the RF-F and $0.69 \pm 0.04 \mathrm{ng} / \mathrm{ml}$ for the RF-AF rats (Fig. 1B); however, a low-amplitude fluctuation was observed in both groups. For the RF-F group a low-amplitude peak coincided with time of expected mealtime and a second peak was observed during the night (ZT17), whereas for the RF-AF group the mealtime-related peak was attenuated and only a main peak was observed during the night (ZT17). Due to the low waveform amplitude, the one-way ANOVA indicated no significant time-related effects for both groups $\left(\mathrm{F}_{(7,41)}=1 \cdot 8\right.$, NS for RF-F and $\mathrm{F}_{(7}$, 40) $=1 \cdot 6$, NS for RF-AF).

Adipose tissue fresh weight No significant oscillations were observed in the wet weight of retroperitoneal and epididymal white adipose tissue. The AL group exhibited in the retroperitoneal $(3.4 \pm 0.2 \mathrm{~g})$ and epididymal fat $(3 \cdot 7 \pm 0 \cdot 2 \mathrm{~g})$ the highest values, which were significantly reduced in RF rats $(1 \cdot 5 \pm 0 \cdot 1$ and $2 \cdot 4 \pm 0 \cdot 1$ g respectively) and remained low in both fasted groups: RF-AF $(1 \cdot 3 \pm 0 \cdot 1$ and $2.4 \pm 0.1 \mathrm{~g})$ and RF-F group $(1.7 \pm 0.1$ and $2 \cdot 7 \pm 0 \cdot 1 \mathrm{~g})$. A positive correlation between leptin concentration and adipose tissue weight was observed among the four groups for epididymal $(r=0 \cdot 8)$ and retroperitoneal fat $(r=0 \cdot 9)$.

Serum FFA In the AL group, serum FFAs exhibited a mean concentration of $8 \cdot 4 \pm 0.3 \mathrm{mg} / \mathrm{dl}$ with a very slight and not significant fluctuation (Fig. 2A). In contrast, RF animals exhibited a higher FFA mean concentration $(11.2 \pm 0.6 \mathrm{mg} / \mathrm{dl})$ with an evident fluctuation, peak levels anticipating mealtime and lowest values after feeding. The two-way ANOVA revealed a significant main effect of group $\left(\mathrm{F}_{(1,111)}=35, P<0 \cdot 001\right)$, time $\left(\mathrm{F}_{(7,111)}=\right.$ $8 \cdot 6, P<0 \cdot 001)$ and group $\times$ time interaction $\left(\mathrm{F}_{(7,111)}=\right.$ $11 \cdot 8, P<0 \cdot 001)$.

During both fasting conditions, mean concentration of FFA was notably increased as compared with $\mathrm{AL}$ and RF groups; mean levels for the RF-F group were $35.1 \pm 1.9 \mathrm{mg} / \mathrm{dl}$ and for the RF-AF $28.8 \pm 1 \mathrm{mg} / \mathrm{dl}$. The anticipatory peak of serum FFA associated with mealtime persisted in the RF-F group after two cycles of total food deprivation (Fig. 2B); however, this persistence was not observed in the RF-AF group, which showed peak values during the night. The one-way ANOVA indicated a significant time effect $\left(\mathrm{F}_{(7,40)}=3 \cdot 4, P<0 \cdot 005\right)$ for the RF-F group but revealed no significant time effect for the RF-AF group $\left(\mathrm{F}_{(7,40)}=1 \cdot 5, \mathrm{NS}\right)$.

Stomach fresh weight Rats fed AL showed a lowamplitude diurnal rhythm with higher values during the

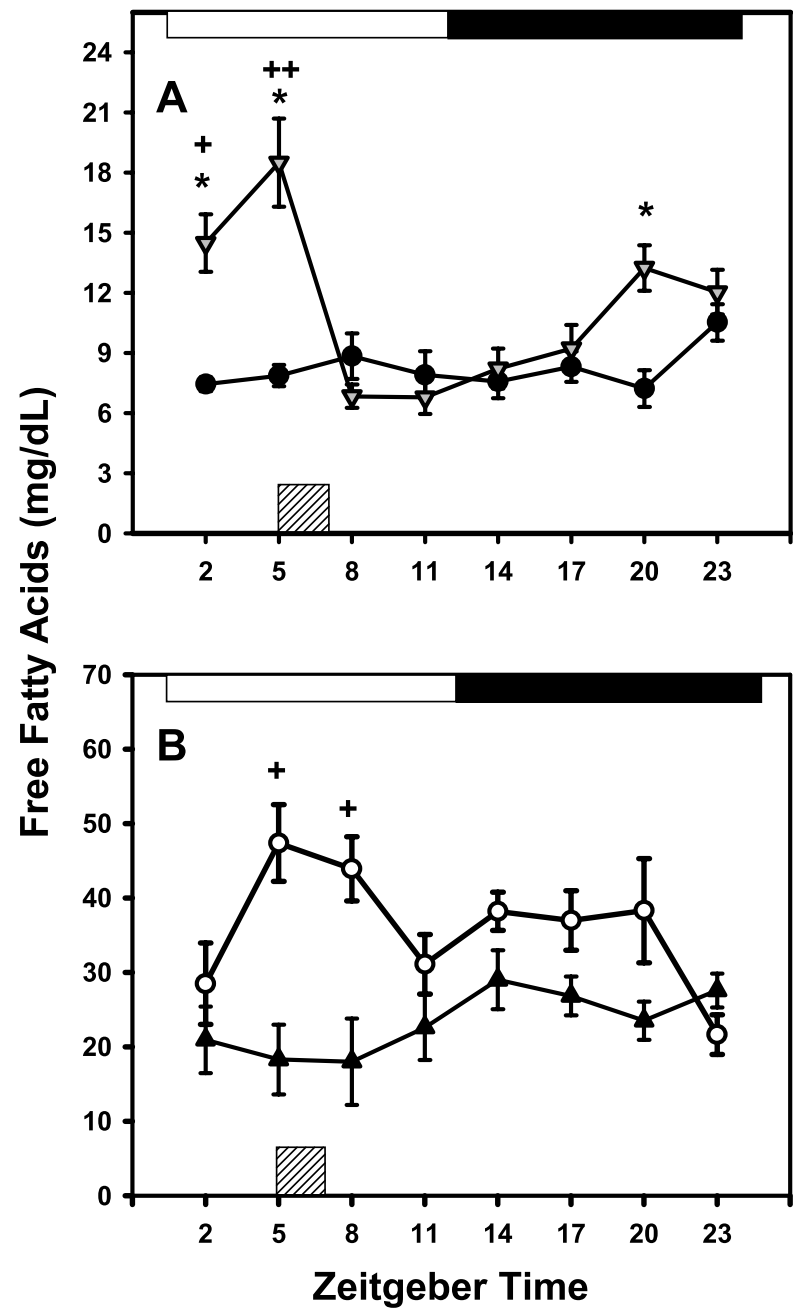

Figure 2 Diurnal rhythms of serum FFA (means \pm S.E.M.), its entrainment by RF (A) and its persistence in fasting conditions (B). In RF rats (gray triangles) a clear anticipatory peak was observed before mealtime. After two cycles of fasting, persistence of the food-entrained rhythm was observed (RF-F, white circles), but not after the refeeding-fasting protocol (black triangles). *Significant difference between $\mathrm{AL}$ (black circles) and RF groups $(P<0 \cdot 0001)$; ${ }^{+}$significant difference in the RF group ZT2 vs ZT8, ZT11 and ZT14 $(P<0 \cdot 01) .{ }^{++}$Significant difference with ZT8 to ZT23 $(P<0 \cdot 01)$. (B) ${ }^{+}$Significant difference from ZT23 in the RF-F group $(P<0 \cdot 02)$. Other indications as in Fig 1.

dark period (ZT14-ZT23). Daily values in AL rats fluctuated between 3.8 and $9.2 \mathrm{~g}$. This diurnal rhythm was entrained by the RFS, producing a high-amplitude oscillation, with lowest values of $2.5 \mathrm{~g}$ before mealtime and highest values of $34 \mathrm{~g}$ after feeding. Visual inspection revealed in RF rats a remarkable capacity for stomach distension during the mealtime followed by a slow and paced emptying velocity that maintained stomach weight significantly higher than $\mathrm{AL}$ for up to $12 \mathrm{~h}$, reaching lowest values only $3 \mathrm{~h}$ before mealtime (Fig. $3 \mathrm{~A}$ ). The 


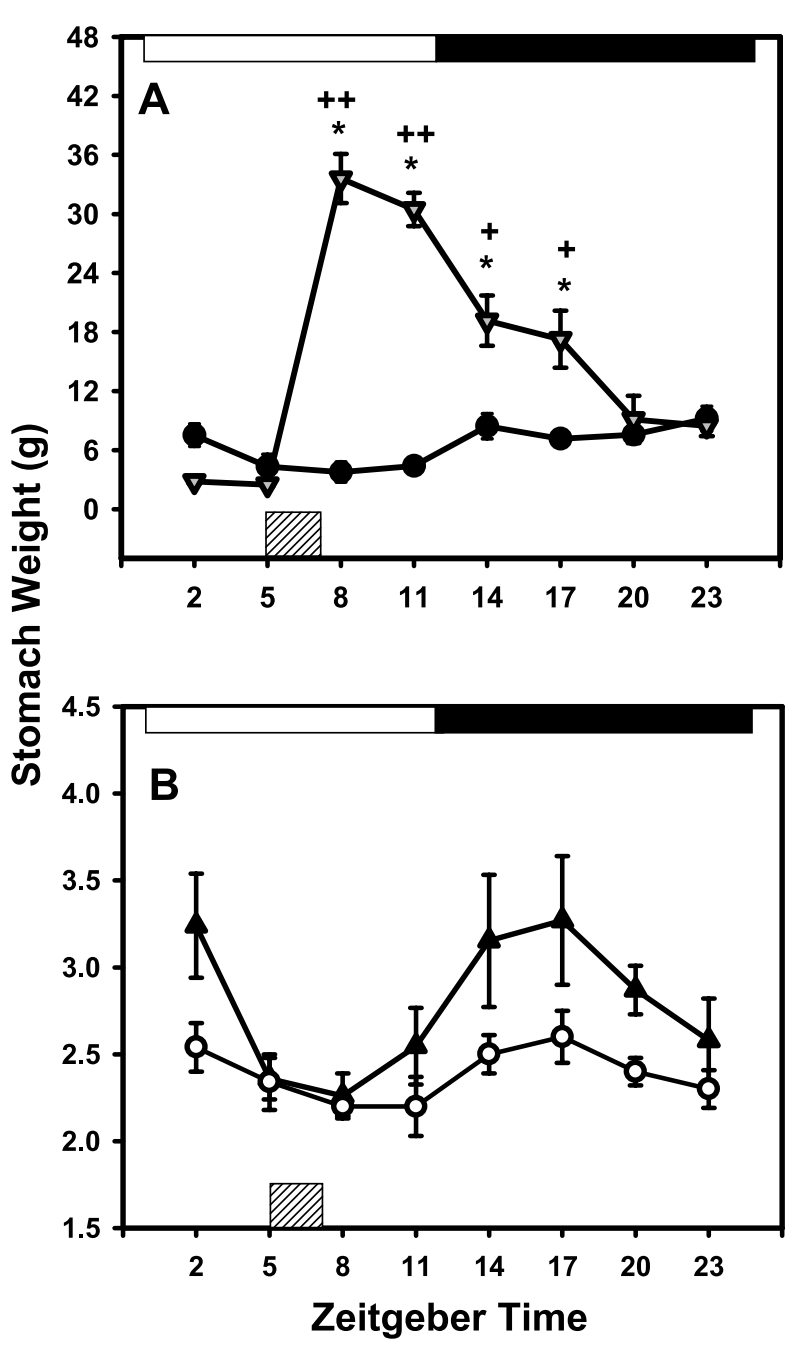

Figure 3 Diurnal rhythms of stomach weight (means \pm S.E.M.), its entrainment by RF (A) and its oscillations in fasting conditions (B). In RF rats (gray triangles), stomach exhibited lowest values before and the highest values after mealtime, followed by a slow decrease along the $22 \mathrm{~h}$ of fasting interval. No persistence of the food-entrained rhythm was observed in fasted groups (RF-F, white circles; RF-AF, black triangles). *Significant difference between $\mathrm{AL}$ (black circles) and RF groups (Tukey, $P<0 \cdot 01$ ). ${ }^{+}$For the RF group significant difference with ZT2, ZT5, ZT20 and ZT23 $(P<0 \cdot 01)$. ${ }^{++}$Significant difference with ZT14, ZT17, ZT20, ZT23, ZT2 and ZT5 (Tukey, $P<0 \cdot 0001$ ). Other indications as in Fig 1.

two-way ANOVA indicated a significant main effect between groups $\left(\mathrm{F}_{(1,48)}=121 \cdot 7, P<0.001\right)$, due to time $\left(\mathrm{F}_{(7,48)}=24 \cdot 8, \quad P<0 \cdot 001\right)$ and group $\times$ time interaction $\left(\mathrm{F}_{(7,48)}=34 \cdot 5, P<0 \cdot 001\right)$.

As expected under fasting conditions, stomach weight was lower than in fed rats at all time points; mean values for both groups fluctuated between 2 and $3.5 \mathrm{~g}$. The food-entrained pattern did not persist in RF-F nor in RF-AF rats. Both fasted groups showed low-amplitude stomach-weight oscillation phases related to the daily cycle and slightly higher values during the night (Fig. 3B). The one-way ANOVA revealed no significant time effect for the RF-F group $\left(\mathrm{F}_{(7,40)}=1 \cdot 5\right.$, NS), but a significant time effect for the RF-AF group $\left(\mathrm{F}_{(7,48)}=2 \cdot 6, P<0 \cdot 02\right)$.

Behavioral data All rats showed a consistent anticipatory drinking behavior (Fig. 4), which started about $1 \mathrm{~h}$ before food access and reached a peak of activation at the moment of food arrival. In both fasting protocols, RF-F and RF-AF rats exhibited persistence of AA during the expected mealtime.

\section{Experiment II}

Rats showed stable free-running rhythms with periods ranging between $24 \cdot 2$ and $24 \cdot 4 \mathrm{~h}$. Leptin injections induced a clear increase in drinking behavior immediately after the i.c.v. administration; however, daily leptin administration did not induce anticipatory drinking activity (Fig. 5), nor did it produce a modification of free-running rhythmicity. Likewise, i.c.v. vehicle administration produced an immediate increase in drinking activity after injection but of lower amplitude. This was further verified with the activity average waveforms, in which a clear activation after leptin and vehicle injection was observed, but no AA nor a difference between groups (Fig. 6). The two-way ANOVA for the $3 \mathrm{~h}$ previous to and $3 \mathrm{~h}$ following the injections did not reveal a significant effect of treatment $\left(\mathrm{F}_{(1,6)}=0 \cdot 06\right.$, NS), but a significant effect of time $\left(\mathrm{F}_{(24,144)}=2 \cdot 3, P<0 \cdot 001\right)$ and no effect of treatment $\times$ time interaction $\left(\mathrm{F}_{(24,144)}=1 \cdot 1, \mathrm{NS}\right)$.

Histological verification confirmed that in the reported animals the tip of the cannula had reached the third ventricle.

\section{Discussion}

Experiment 1 demonstrated that in AL conditions leptin, FFA and stomach weight exhibit daily rhythms adjusted to the light-darkness cycle. When food availability is restricted to $2 \mathrm{~h}$ daily, diurnal rhythms are entrained and shift their phase around mealtime. These food-entrained rhythms persisted for two cycles in fasting conditions; however, after an AL-fasting procedure, rhythmicity was completely phase adjusted to the light-darkness cycle. For the stomach weight this shift to the light-darkness cycle was evident even after two cycles of fasting. In addition, leptin and FFA exhibited an adjustment in their mean concentration levels according to the metabolic state in each group, indicating a simultaneous expression of a homeostatic and a circadian regulatory process.

The finding that leptin rhythm is entrained by mealtime is in agreement with a previous report in mice (Ahima et al. 1998). Likewise, the peak in FFA levels in anticipation to food access confirms previous results reported by our group (Escobar et al. 1998). The present study provides 

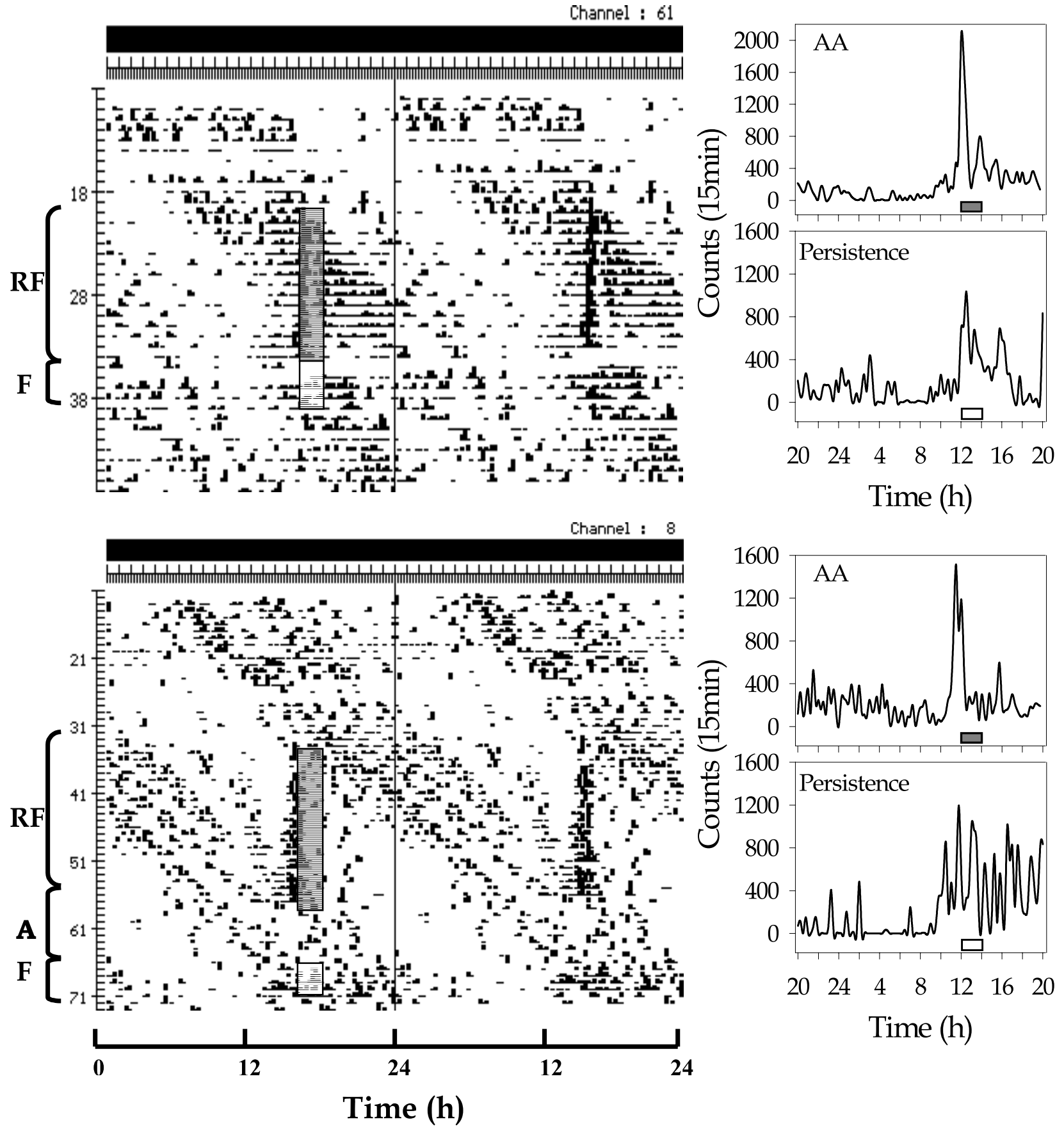

Figure 4 Double-plotted actograms and activity waveforms of drinking behavior of two representative rats recorded under the two experimental conditions. Top panel shows activity of one RF-F rat. Bottom panel shows activity of an RF-AF rat. In both actograms and activity waveforms, the gray bar indicates time of food access during RFS, and the white bar indicates the expected mealtime during fasting conditions. In both cases, animals exhibit robust AA during the RFS and persistence in fasting conditions. For the RF-F group, data were lost during the first 2 days (days 34-35) of fasting conditions, due to technical problems.

evidence that these entrained rhythms persist only for a few cycles after discontinuing the RFS and adjust primarily to the light-darkness cycle, which confirms the pre- dominant influence of the SCN on peripheral organs and metabolic rhythms (Buijs et al. 2003). A recent study reported a similar shift to the light-darkness cycle in 


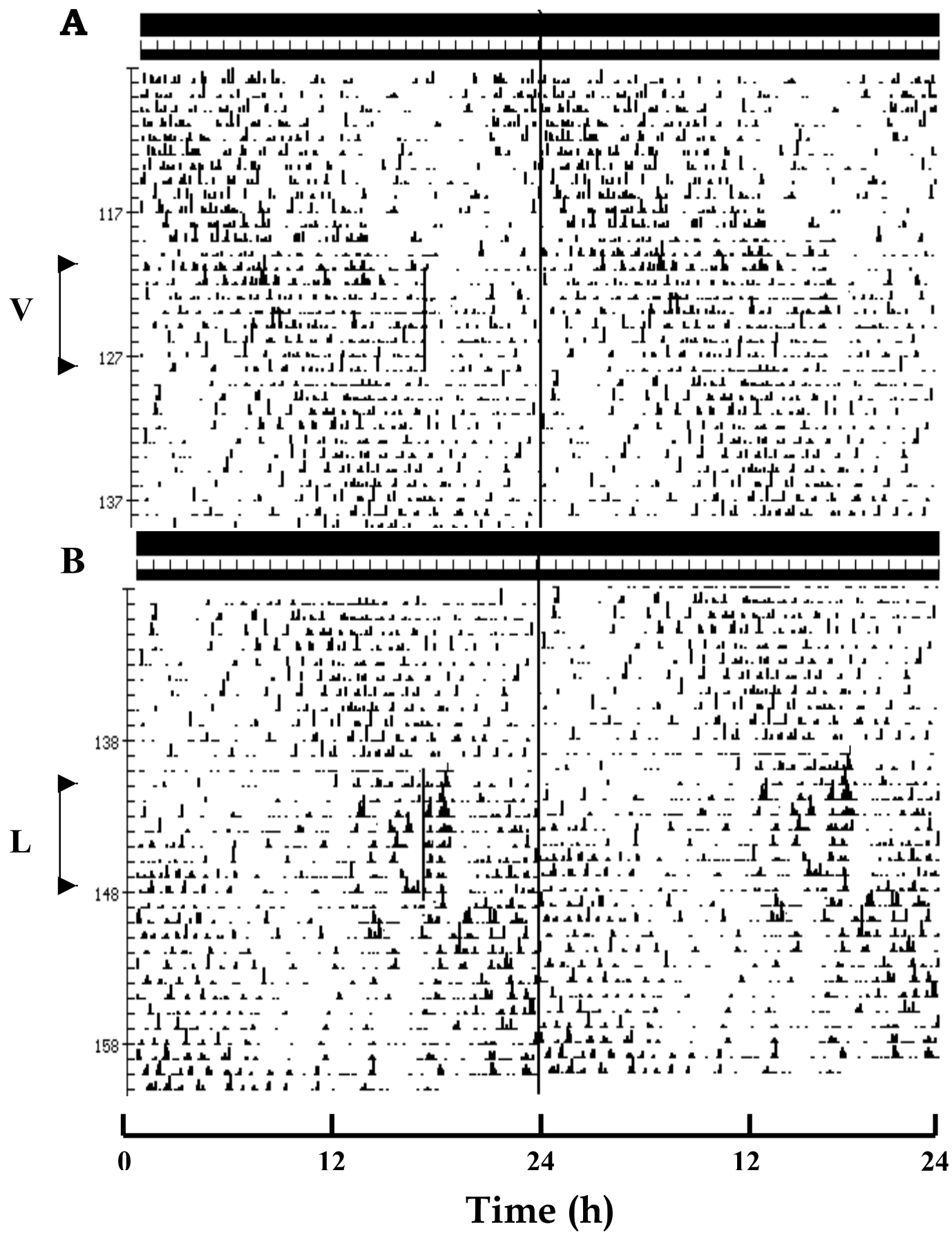

Figure 5 Representative double-plotted actograms of drinking behavior from animals receiving a daily i.c.v. injection with vehicle (A) or leptin (B). The arrows indicate the 10 day interval of the injections. The line indicates time of administration (14 $00 \mathrm{~h})$. $\mathrm{V}=$ vehicle, $\mathrm{L}=$ leptin administration.

gastrointestinal tract and liver clock protein Per1 foodentrained rhythms when rats were left in ad libitum conditions (Davidson et al. 2003). These molecular and metabolic findings contrast with the behavioral anticipatory response and its persistence in fasting conditions here reported and previously described by other groups (Mistlberger 1994, Stephan 2001). These observations together suggest that the mechanisms underlying metabolic entrainment are different from those underlying behavioral and locomotor food AA. 


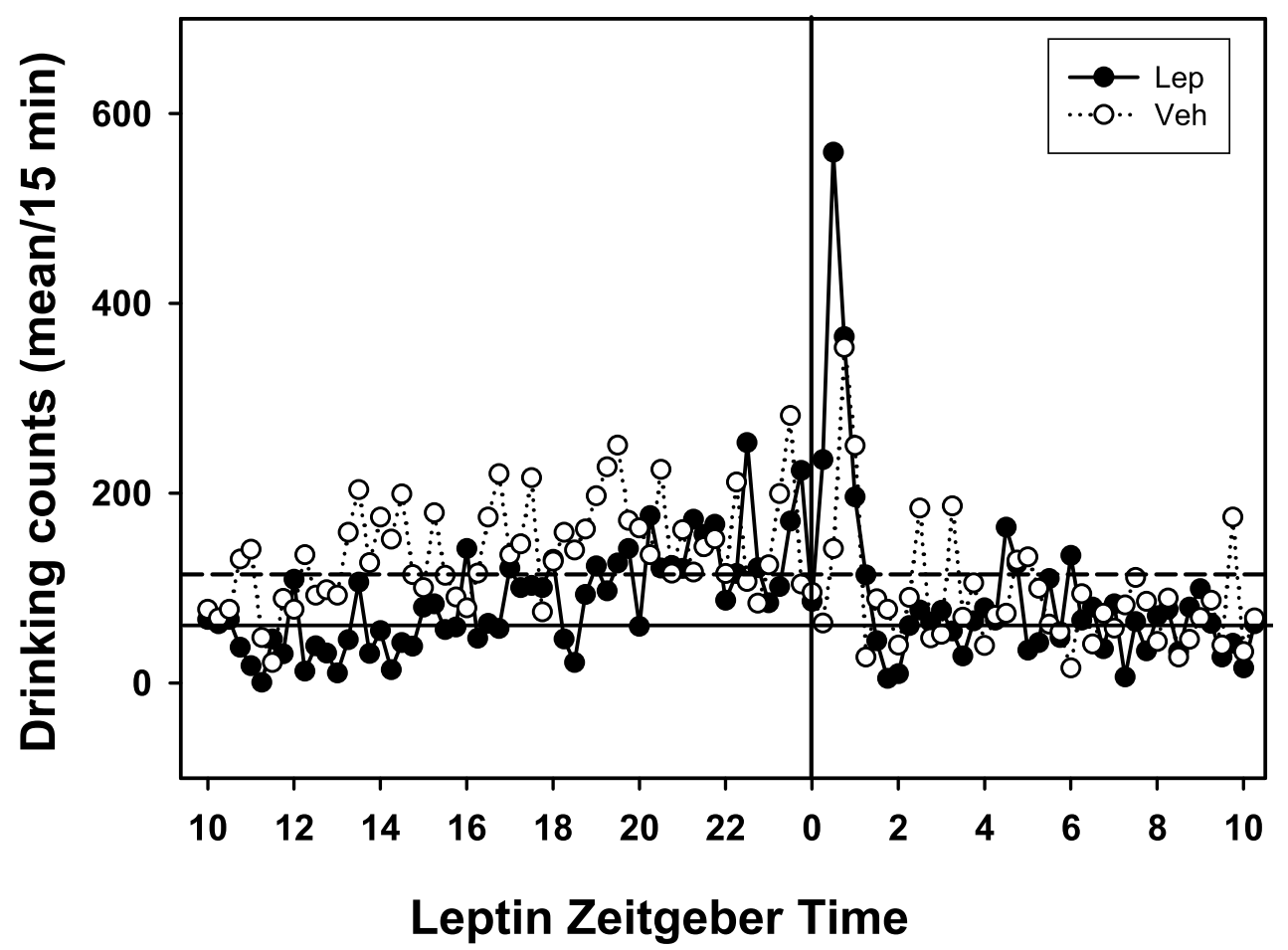

Figure 6 Mean drinking waveform in 15 min time bins from animals exposed to daily i.c.v. leptin (Lep) or vehicle (Veh) administration. The vertical line shows time of administration (1400 h) and the horizontal lines show the mean basal activity for each group.

Stomach weight in RF rats showed a remarkable filling and distension capacity and a slow paused emptying that lasted up to $12 \mathrm{~h}$ leading to an empty stomach only $3 \mathrm{~h}$ prior to food access. Under an RF protocol, rats ingested during the short $2 \mathrm{~h}$ interval a similar amount of food as the $\mathrm{AL}$ rats (data not shown). For a different set of rats we have estimated a daily ingestion of $23-25 \mathrm{~g}$ for $\mathrm{AL}$ and of 21-24 $\mathrm{g}$ in RF rats. This adaptation process of the stomach allows storing large quantities of food and may provide continuous nutrient supply to the organism in spite of the $22 \mathrm{~h}$ fasting interval. This same dynamics in stomach distension was previously reported in rats, although not with a circadian approach (Lima et al. 1981, Curi et al. 1984), and in young rabbits that are lactated once a day and attain a substantial stomach distension followed by a slow emptying rate (Escobar et al. 2000). Because this apparent food-entrained rhythm shifted its phase to the light-darkness cycle even after one cycle in ad libitum conditions, we assume that it reflects a direct response to feeding. It is well reported that the distension and emptying of the stomach produces peptides that transmit signals to the nervous system (Ueta et al. 1991, Horvath et al. 2001, Hewson et al. 2002). Thus, it remains to be established whether the distension or slow emptying of the stomach may constitute a time signal for the FEO.

In experiment 2, daily i.c.v. leptin administration did not produce drinking AA. The mean activity profile indicated an intense response of drinking behavior $30 \mathrm{~min}$ after leptin administration followed by behavioral inhibition. The initial assessment of food ingestion indicated a consistent inhibitory effect on food ingestion, as previously reported (Campfield et al. 1995, Flynn et al. 1998, Wang et al. 1999), and allowed us to presume that injected leptin produced the expected effect on hypothalamic structures. Although the literature provides evidence that leptin may be a peripheral signal to the hypothalamic nuclei (Hewson et al. 2002), daily leptin administration did not produce FAA. Thus we may assume that leptin is not the internal entraining signal to central elements of the FEO.

The lack of effects due to daily leptin administration also may be explained by the fact that animals were fed ad libitum and under this condition the FEO is not expressed. In a previous study we reported that daily i.p. adrenaline administration produced AA in free-feeding rats (Mendoza et al. 2003). However, it is possible that adrenaline produced the catabolic state necessary for the expression of the FEO trough glycolysis and FFA mobilization, while leptin i.c.v. injection did not directly affect peripheral metabolism. On the other hand it is possible that the time of leptin administration could have coincided with decreased hypothalamic leptin receptor sensitivity, which may lead to a diminished response to leptin during the day (Xu et al. 1999, Brogan et al. 2000, Denis et al. 2003). In this study it is difficult to define this temporal 
relationship because animals were free-running in constant darkness and to our knowledge there is no report of free-running rhythms for leptin or its receptors.

Although our data do not support a relationship of leptin with the FEO's entraining pathway, it has been reported that it may have a strong link with the SCN. Leptin levels are modulated by the SCN, and in the rat SCN lesions abolish its diurnal rhythmicity (Kalsbeek et al. 2001). A recent study described phase advances in neuronal activity in a dose-dependent manner produced by leptin in brain slices containing the SCN (Prosser \& Bergeron 2003), suggesting that leptin may modulate the SCN phase.

In conclusion, the present results provide evidence that adipose tissue metabolic signals such as leptin and FFA exhibit oscillations associated with mealtime. Only FFA food-entrained rhythms persist for a few cycles in fasting. Therefore we assume that metabolic rhythms respond to feeding and are mainly locked to the light-darkness cycle. In contrast, AA persists even after nine or ten cycles in fasting. These observations together suggest that metabolic entrainment and behavioral or locomotor food AA depend on different regulatory mechanisms. Because daily i.c.v. administration of leptin did not promote AA, we conclude that leptin is not mediating an endogenous entraining signal for the FEO. It remains to be established to what extent the dynamics of the extreme stomach distension or its complete emptying may be associated with the entraining signal for this oscillator.

\section{Acknowledgements}

The authors wish to thank Dr Ruud Buijs, The Netherlands Institute for Brain Research critical comments on the manuscript.

\section{Funding}

This study was supported by grants DGAPA-UNAM-IN204800 and CONACyT 33033-N to C E.

\section{References}

Ahima RS 2000 Leptin and neuroendocrinology of fasting. Frontiers of Hormones Research 26 42-56.

Ahima RS \& Flier JS 2000 Adipose tissue as an endocrine organ. Trends in Endocrinology and Metabolism 11 327-332.

Ahima RS, Prabakaran D \& Flier JS 1998 Postnatal leptin surge and regulation of circadian rhythm of leptin by feeding: implications for energy homeostasis and neuroendocrine function. Journal of Clinical Investigation 101 1020-1027.

Aguilar-Roblero R, Salazar-Juárez A, Rojas-Castañeda J, Escobar C \& Cintra 1997 Organization of circadian rhythmicity and suprachiasmatic nuclei in malnourished rats. American Journal of Physiology. Regulatory, Integrative and Comparative Physiology 42 R1321-R1331.
Bartness TJ 1987 Animal and human fat changes: measurement and interpretation. In Feeding and Drinking, pp 463-498. Eds FM Toates \& NE Rowland. New York and Amsterdam: Elsevier Science Publishers.

Brogan RS, Grove KL \& Smith MS 2000 Differential regulation of leptin receptor but not orexin in the hypothalamus of the lactating rat. Journal of Neuroendocrinology 12 1077-1086.

Buijs RM, van Eden CG, Goncharuk VD \& Kalsbeek A 2003 The biological clock tunes the organs of the body: timing by hormones and the autonomic nervous system. Journal of Endocrinology 177 17-26.

Buyse M, Bado A \& Dauge V 2001 Leptin decreases feeding and exploratory behaviour via interactions with $\mathrm{CCK}_{1}$ receptors in the rat. Neuropharmacology 40 818-825.

Campfield LA, Smith FJ, Guisez Y, Devos R \& Burn P 1995 Recombinant mouse $\mathrm{OB}$ protein: evidence for a peripheral signal linking adiposity and central neural networks. Science 269 546-549.

Curi R, Hell NS, Bazotte RB \& Timo-Iaria 1984 Metabolic performance of free fed rats subjected to prolonged fast as compared to the metabolic pattern in rats under long term food restriction. Physiology and Behavior 33 525-531.

Dallongeville J, Hecquet B, Lebel P, Edme JL, Le Fur C, Fruchart JC, Auwerx J \& Romon M 1998 Short term response of circulating leptin to feeding and fasting in man: influence of circadian cycle. International Journal of Obesity 22 728-733.

Damiola F, Le Minh N, Preitner N, Kornmann B \& Fleury-Olela F 2000 Restricted feeding uncouples circadian oscillators in peripheral tissues from the central pacemaker in the suprachiasmatic nucleus. Genes and Development 14 2950-2961.

Davidson AJ \& Stephan FK 1998 Circadian food anticipation persists in capsaicin deafferented rats. Journal of Biological Rhythms $\mathbf{1 3}$ 422-429.

Davidson AJ \& Stephan FK 1999 Plasma glucagon, glucose, insulin, and motilin in rats anticipating daily meals. Physiology and Behavior 66 309-315.

Davidson AJ, Poole AS, Yamazaki S \& Menaker M 2003 Is the food-entrainable circadian oscillator in the digestive system? Genes, Brain and Behavior 2 32-39.

Denis RGP, Bing C, Naderali EK \& Vernon RG 2003 Lactation modulates diurnal expression profiles of specific leptin receptor isoforms in the rat hypothalamus. Journal of Neuroendocrinology 178 225-232.

Díaz-Muñoz M, Vázquez MO, Aguilar-Roblero R \& Escobar C 2000 Anticipatory changes in liver metabolism and entrainment of insulin, glucagon, and corticosterone in food-restricted rats. American Journal of Physiology. Regulatory, Integrative and Comparative Physiology 279 R2048-R2056.

Elimam A \& Marcus C 2002 Meal timing, fasting and glucocorticoids interplay in serum leptin concentrations and diurnal profile. European Journal of Endocrinology 147 181-188.

Elmquist JK 2001 Hypothalamic pathways underlying the endocrine, autonomic, and behavioral effects of leptin. Physiology and Behavior 74 703-708.

Escobar C, Díaz-Muñoz M, Encinas F \& Aguilar-Roblero R 1998 Persistence of metabolic rhythmicity during fasting and its entrainment by restricted feeding schedules in rats. American Journal of Physiology. Regulatory, Integrative and Comparative Physiology 274 R1309-R1316.

Escobar C, Hudson R, Martínez-Gómez \& Aguilar-Roblero R 2000 Metabolic correlates of the circadian pattern of suckling-associated arousal in young rabbits. Journal of Comparative Physiology A 186 33-38.

Flynn MC, Scott TR, Pritchard TC \& Plata-Salamán CR 1998 Mode of action of $\mathrm{OB}$ protein (leptin) on feeding. American Journal of Physiology. Regulatory, Integrative and Comparative Physiology 44 R174-R179.

Friedman JM 2000 Obesity in the new millennium. Nature $\mathbf{4 0 4}$ 632-634. 
Halaas JL, Boozer C, Blair-West J, Fidahusein N, Denton DA \& Friedman JM 1997 Physiological response to long-term peripheral and central leptin infusion in lean and obese mice. PNAS $\mathbf{9 4}$ 8878-8883.

Hewson AK, Tung LYC, Tookman L \& Dickson SL 2002 The rat arcuate nucleus integrates peripheral signals provided by leptin, insulin, and ghrelin mimetic. Diabetes 51 3412-3419.

Honma KI, Honma S \& Hiroshige T 1984 Feeding-associated corticosterone peak in rats under various feeding cycles. American Journal of Physiology. Regulatory, Integrative and Comparative Physiology 246 R721-R726.

Honma S, Honma KI, Nagasaka T \& Hiroshige T 1987 The ventromedial hypothalamic nucleus is not essential for the prefeeding corticosterone peak in rats under restricted daily feeding. Physiology and Behavior 39 211-215.

Horvath TL, Diano S, Sotonyi P, Heiman M \& Tschop M 2001 Minireview: ghrelin and the regulation of energy balance - a hypothalamic perspective. Endocrinology 142 4163-4169.

Kahler A, Geary N, Eckel LA, Campfield LA, Smith FJ \& Langhans 1998 Chronic administration of OB protein decreases food intake by selectively reducing meal size in male rats. American Journal of Physiology. Regulatory, Integrative and Comparative Physiology 275 R180-R185.

Kalsbeek A, Fliers E, Romjn JA, La Fleur SE, Wortel J, Bakker O, Endert E \& Buijs RM 2001 The suprachiasmatic nucleus generates the diurnal changes in plasma leptin levels. Endocrinology 142 $2677-2685$.

Krieger DT 1974 Food and water restriction shifts corticosterone, temperature, activity and brain amine periodicity. Endocrinology 95 1195-1201.

Krieger DT, Hauser H \& Krey LC 1977 Suprachiasmatic nuclear lesions do not abolish food-shifted ciradial adrenal and temperature rhythmicity. Science 197 398-399.

Lima FB, Hell NS, Timo-Iaria C, Scivoletto R, Dolnikoff MS \& Pupo AA 1981 Metabolic consequences of food restriction in rats. Physiology and Behavior 27 115-123.

Mendoza JY, Aguilar-Roblero R, Díaz-Muñoz M \& Escobar C 2003 Daily epinephrine but not norepinephrine administration produces anticipatory drinking behavior in rats. Biological Rhythms Research 34 73-90.

Mistlberger RE 1994 Circadian food-anticipatory activity: formal models and physiological mechanisms. Neuroscience Behavior Review 18 171-195.

Nóvák M 1965 Colorimetric ultramicro method for the determination of free fatty acids. Journal of Lipid Research 6 431-433.
Paxinos G \& Watson C 1986 The Rat Brain in Stereotaxic Coordinates. New York: Academic Press.

Prosser RA \& Bergeron HE 2003 Leptin phase-advances the rat suprachiasmatic circadian clock in vitro. Neuroscience Letters 336 139-142.

Ruffin MP \& Nicolaidis S 2000 Intracerebroventricular injection of murine leptin enhances the postprandial metabolic rate in the rat. Brain Research 874 30-36.

Schwartz MW, Woods SC, Porte D Jr, Seeley RJ \& Baskin DG 2000 Central nervous system control of food intake. Nature 404 661-671.

Sinha MK, Ohannesian JP, Heiman ML, Kriauciunas A, Stephens TW, Magosin S, Marco C \& Caro JF 1996 Nocturnal rise of leptin in lean, obese, and non-insulin-dependent diabetes mellitus subjects. Journal of Clinical Investigation 97 1344-1347.

Stephan FK 2001 Food-entrainable oscillators in mammals. In Handbook of Behavioral Neurobiology. Circadian Clocks, vol 12, pp 223-246. Eds JS Takahashi \& FW Turek. New York: Kluwer Academic/Plenum Publishers.

Stephan FK 2002 The 'other' circadian system: food as a zeitgeber. Journal of Biological Rhythms 17 284-292.

Terazono H, Mutoh T, Yamaguchi S, Kabayashi M, Akiyama M, Udo R, Ohdo S, Okamura H \& Shibata S 2003 Adrenergic regulation of clock gene expression in mouse liver. PNAS $\mathbf{1 0 0}$ 6795-6800.

Ueta Y, Kannan H \& Yamashita H 1991 Gastric afferents to the paraventricular nucleus in the rat. Experimental Brain Research $\mathbf{8 4}$ 487-494.

Wang T, Hartzell DL, Rose BS, Flatt WP, Hulsey MG, Menon NK, Makula RA \& Baile CA 1999 Metabolic responses to intracerebroventricular leptin and restricted feeding. Physiology and Behavior 65 839-848.

Xu B, Kalra PS, Farmerie WG \& Kalra SP 1999 Daily changes in hypothalamic gene expression of neuropeptide Y, galanin, proopiomelanocortin, and adipocyte leptin gene expression and secretion: effect of food restriction. Endocrinology 140 2868-2875.

Zhang Y, Proenca R, Maffei M, Barone M, Leopold L \& Friedman JM 1994 Positional cloning of the mouse obese gene and its human homologue. Nature 372 425-432.

Received 8 October 2003

Accepted 15 December 2003

Made available online as an

Accepted Preprint 29 December 2003 